\title{
Computational Micromechanics of Fatigue of Microstructures in the HCF-VHCF Regimes
}

\author{
Gustavo M. Castelluccio ${ }^{1}$, William D. Musinski ${ }^{2}$, and David L. McDowell ${ }^{3, *}$ \\ ${ }^{1}$ Sandia National Laboratories, Albuquerque, NM, USA \\ ${ }^{2}$ Materials and Manufacturing Directorate, Air Force Research Laboratory, Wright-Patterson AFB, OH, \\ USA \\ ${ }^{3}$ Georgia Institute of Technology, Atlanta, GA, USA
}

\begin{abstract}
Advances in higher resolution experimental techniques have shown that metallic materials can develop fatigue cracks under cyclic loading levels significantly below the yield stress. Indeed, the traditional notion of a fatigue limit can be recast in terms of limits associated with nucleation and arrest of fatigue cracks at the microstructural scale. Although fatigue damage characteristically emerges from irreversible dislocation processes at sub-grain scales, the specific microstructure attributes, environment, and loading conditions can strongly affect the apparent failure mode and surface to subsurface transitions. In this paper we discuss multiple mechanisms that occur during fatigue loading in the high cycle fatigue (HCF) to very high cycle fatigue (VHCF) regimes. We compare these regimes, focusing on strategies to bridge experimental and modeling approaches exercised at multiple length scales and discussing particular challenges to modeling and simulation regarding microstructure-sensitive fatigue driving forces and thresholds. We conclude by discussing some of the challenges in predicting the transition of failure mechanisms at different stress and strain amplitudes.
\end{abstract}

Keywords: Very High Cycle Fatigue, Failure Mechanisms, Microstructure Effects, Mesoscale Modeling

1. Introduction

Historically, fatigue life estimations have been loosely classified into categories of low cycle fatigue (LCF) and high cycle fatigue (HCF), which have respectively been associated with fatigue lives that are typically on the order of $10^{3}$ and $10^{6}$ cycles for structural steels. This classification roughly demarcates fatigue processes according to dominance of macroscopic cyclic plastic deformation relative to elastic deformation (LCF), and vice versa (HCF). The advent of higher fidelity experimental methods (e.g., in situ microscopy and digital image correlation) has provided evidence that fatigue cracks form at stress levels significantly below the macroscopic yield strength, for which fatigue lives can extend well beyond $10^{6}$ cycles. The very high cycle fatigue (VHCF) and ultra high cycle fatigue (UHCF) regimes typically refer to fatigue failure beyond $10^{9}$ and $10^{12}$ loading cycles, respectively. These regimes have become increasingly studied from an experimental perspective over the past two decades, owing largely to the pioneering efforts of Prof. Claude Batthias, to whom this article gives tribute [1], [2]. It is practical from an

*Corresponding author. Email: david.mcdowell@me.gatech.edu 
engineering perspective to classify fatigue damage processes in terms of fatigue life; however, the applicability of physically-based fatigue models should be assessed in terms of the operative failure mechanisms. In fact, it is of urgent interest to explore and understand mechanisms and the roles of polycrystallinity and other attributes of microstructure that give rise to distinctions in heuristics of fatigue lifing algorithms through LCF to HCF to VHCF regimes.

Along these lines, it is first pertinent to mention the so-called fatigue limit [1], which refers to the maximum cyclic stress amplitude at which fatigue failure is not observed the specimen scale for a given load ratio. Due to prior limitations in testing capabilities (frequency and required test time) this fatigue limit was traditionally attributed to a stress amplitude that a component would withstand over $10^{6}$ or $10^{7}$ fatigue cycles. It is known [3] that fatigue cracks can form below this limit, but can then be arrested under constant amplitude loading for certain alloys. For others, successively reduced amplitudes of loading in the HCF and even VHCF regimes show no evidence of an arrest limit [2], but are observed to result in failure mode transitions from surface to subsurface crack initiation. Beyond this traditional fatigue limit, in the VHCF regime and beyond, cracks can still form within slip bands or via other mechanisms such as subsurface particle decohesion [2]-[4] provided that a threshold driving force for activation of these mechanisms is exceeded. Consequently, the activation of multiple mechanisms [5] can induce a change in the S-N curve shape (e.g., multiple stages [6]). This has led to important consequences in terms of enlightened understanding of the notion of fatigue limit, as well as its limitations.

Current technology has not yet reached agreement regarding the formulation of mesoscale driving forces necessary to address sub-micron mesoscale phenomena in the formation of fatigue cracks (e.g., details of dislocation structures during strain localization, the role of free surfaces, environmental ingress, etc.). Therefore, approximate continuum models for fatigue driving forces that serve as surrogate measures for subscale processes are employed to compare the fatigue resistance of various microstructures obtained through different thermomechanical process route, loading conditions and histories. However, these surrogate measures rely on "top-down" experimental work that characterizes failure mechanisms. Since the origin of irreversible cyclic deformation in dislocation networks and at interfaces is coupled with microstructure morphology, it is necessary to parlay this understanding into modeling strategies. In simple terms, this requires the introduction of fatigue assessments that compute state variables for evolving microstructure and fatigue indicator parameters (FIPs) that correlate with progressive fatigue damage over a certain volume that serves to describe a damage process zone (DPZ). These FIPs serve as proxies for the driving force to form and grow microstructurally small fatigue cracks within the DPZ, and depend on the failure mechanism (i.e., they may differ for HCF or VHCF).

In this paper we discuss the effects of microstructure on fatigue failure in the HCF and VHCF regimes. Although the focus is on structural metallic materials in ambient environment, some of the aspects discussed can be extrapolated to nanomaterials and extreme applications. Some of this understanding is gleaned from experiments and some from accompanying computational modeling and simulation. Over the next sections we briefly explore the relation of fatigue damage as a function of strain 
amplitude and the importance of microstructure attributes on fatigue resistance. We discuss appropriate modeling strategies and challenges/limitations to quantify fatigue driving forces over microstructure length scales and key differences between HCF/VHCF regimes. In addition, we discuss effects of microstructure morphology on fatigue and challenges in the integration of models and experiments.

2. Interpretation of damage mechanisms in the HCF and VHCF Regimes

Significant experimental evidence has shown that fatigue behavior differs in the HCF and VHCF regimes, even though fatigue cracks form in both regimes as a fundamental consequence of irreversibility of cyclic rearrangement of a dislocation network in embryonic crack formation and early growth stages. Under HCF, cracks often form on the surface due to the formation of extrusions or decohesion of grain boundaries. On the contrary, under VHCF cracks tend to form subsurface and produce a "fish eye" fracture surface [2]. Once this crack reaches the surface, there is a rapid transition to different fatigue crack growth rates and morphologies. The classification of fatigue damage mechanisms based on the number of cycles to failure is often vague and ambiguous. This underlines the need to characterize the distinctive physics underlying fatigue cracks that emanate from the surface versus the bulk of the material.

Free surfaces provide the opportunity to relieve stresses that promote highly localized strain. Surface crack formation occurs due to the localization of strain coupled with an environmental enhancement of mechanical irreversibility. Foreign species diffuse preferentially along fast paths (highly localized strained areas, grain boundaries, etc.), driven by either a difference in chemical potential or transported by the core of dislocations. Subsurface crack formation often occurs at discontinuities such as voids, inclusions or secondary phases, with much lower influence of the environment [7]. Whether a crack will fail from a surface or subsurface initiation site is therefore related to both the probability of occurrence of microstructure discontinuities at and below the surface and the degree to which the environment enhances surface crack propagation [8].

Microstructure attributes such as the size and morphology of grains, texture, number and location of discontinuities, inclusions, and second phases determine the likelihood of developing crystallographic crack embryos that can eventually intensify the local favorable stress concentration. Furthermore, the local stress intensification depends not only on the geometrical attributes of one grain, but also on the characteristics of the neighboring grains. Indeed, first and second nearest neighbor grains can be crucial in inducing fatigue damage and determining the fate of a particular microstructure arrangement [9]. This explains why seemingly identical inclusions, pores or grains might lead to early failure or no fatigue cracks at all.

The sources or mechanisms of dominant irreversibility depend on the amount of deformation (or energy) introduced into the system. The irreversible deformation of structural ductile metals with grain size above one micron in the HCF regime tends to be dominated by the net dislocation accumulation in each loading cycle. As-produced dislocation structures in metals evolve to yield cyclic-efficient structures that reduce the energy consumed per cycle. In HCF, this is observed in the reduction of the energy 
consumed per cycle (known as loop shape factor $V_{H}$ ) with decreasing applied strain [10]; the evolution of the loop shape factor correlates with the formation of persistent slip bands in wavy slip metals. For higher applied strains (typically LCF), there is almost no change of $\mathrm{V}_{\mathrm{H}}$. In the case of low stacking fault energy (SFE) metallic materials under HCF, slip is controlled by dislocation pile-ups due to limited cross slip (though it depends on the peak stress and temperature). In addition, the introduction of twins and stressinduced phase transformation such as martensite increase the deformation anisotropy. These effects are coupled with the diffusion of species, orientation of the crystal lattice, presence of second phases and morphology of grain boundaries. For example, Matsunaga and Noda [11] showed that the formation of $\varepsilon$-martensite in austenitic strainless steels controlled the diffusion of hydrogen, which itself affect slip planarity [12].

In most ductile metallic materials, irreversible mechanisms in the HCF regime are dominated by the net production of dislocations in the bulk of grains, which leads to slip localization and increasing mechanical reversibility. In this case, the deformation energy provided per cycle is sufficient to activate irreversible collective mechanisms in the bulk of at least some grains. Furthermore, the driving force to form a fatigue crack depends not only on the crystallographic direction (e.g., apparent Schimd factor), but also on the deformation and rotation of a cluster of neighbor grains. Indeed, second nearest neighbor grains and beyond affect the formation of transgranular fatigue cracks [9]. Since a typical geometricallysmooth component can have a large number of clusters of grains, fatigue life is driven by the extreme microstructure configurations of the microstructure and the arrangement of clusters of, potentially, hundreds of grains. This has been clearly observed for copper with ultra fine grains, in which the formation of shear bands and fatigue cracks in the HCF to VHCF regimes require the cooperation of multiple grains [13]-[15]. Thus, the mere existence of a stress concentrator such as a material or geometric discontinuity might not be enough to supersede the chances of finding a cluster of contiguous grains that are oriented to be particularly detrimental. Accordingly, the microstructure plays a key role in HCF and VHCF.

Grain boundary fatigue decohesion or intergranular failure via the Stroh-Zener mechanism [16] can coexist and compete with transgranular failure. Under the Stroh-Zener mechanism, the fatigue driving force depends on the accumulated strain impinging a grain boundary and the stress normal to the boundary. The grain boundary character (tilt/twist) influences the ability of dislocations to cross the boundaries and alleviate the driving forces. Although the understanding of dislocation slip transfer across boundaries is still underdeveloped, it is accepted that the blockage of dislocations can induce local stress intensification and pileups, which can lead to boundary decohesion. In this case, production and annihilation of dislocations still control fatigue failure.

In the transition to the VHCF regime, the driving force for fatigue crack formation is reduced, irreversible mechanisms may not be activated in the entire grain, and there is reduced coupling among different "sectors" or domains of each grain. Thus, irreversible deformation depends strongly on the details of the microstructure morphology. For example, triple point junctions, second phases, voids, and inclusions control the formation of embryonic cracks. 
The HCF and VHCF regimes also differ in terms of the dislocation structures developed under cycling loading. The to/fro cyclic slip in HCF usually stimulates organization of dislocations into localized structures (e.g. dislocation ladder structures or cells) that tend to extend over the entire grain [17]. Stress gradients within grains may induce a range of mesoscale dislocation structures with some variations in size. In the VHCF regime, slip bands may form in metals such as copper [4], [18], pure nickel [19], and Nibase superalloys [19] but dislocation walls seem to be thinner, more sparsely distributed, and less organized (e.g., elongated cells rather than perfectly organized ladder type). Dislocation processes (such as multiplication and cross slip) are less collectively organized and occur intensely only at a local scale in the neighborhood of a stress intensification. Indeed, irreversible processes initiate from local stress concentration and plastic strain is highly anisotropic, with strong gradients.

Although dislocation structures are not completely understood in the VHCF regime, some experimental evidence has shown the formation of nanoscale structures that progress along planes of maximum driving force [20]. Weidner et al. [18] have shown evidence of localized shear deformation along ladder-like slip lamella in polycrystalline copper even below the persistent slip band threshold. For single crystal microstructures, in the absence of grain boundaries, other microstructure barriers act as local stress concentrators. For example, in single crystal Ni-base superalloys, cracks can form at microstructure stress concentrators such as carbides or $\gamma / \gamma^{\prime}$ eutectic regions [21] in the VHCF regime. Thus, without geometrical microstructure features such as grain boundaries, precipitates or voids, intrinsic dislocation irreversibility may be insufficient to localize and form cracks; this characteristic effectively pertains to the existence of an intrinsic fatigue limit for these materials.

\section{Dominance of microstructure effects in the HCF and VHCF regimes}

Low applied stress (or strain) amplitudes shift fatigue lives from the LCF to the VHCF regime by reducing mechanical irreversibility. In the absence of sufficient driving force to form and propagate surface cracks in the VHCF regime, more slowly propagating internal cracks tend to be the source of failure. Although subsurface crack formation is not usually strongly affected by the environment [7], foreign species may play a role after long periods or under high diffusivity. For example, studies [22], [23] have linked subsurface fatigue cracking to the concentration of hydrogen. The competition of these mechanisms combined with potency of bulk sites for crack formation such as large grains, inclusions or second phases, form the bases for the experimental observation of surface/subsurface crack formation and the overlapping observations of surface/subsurface crack formation in the transition from HCF to VHCF [24], [25].

Experimental data have shown that higher cyclic stress amplitudes reduce fatigue life variability. In the transition regime from HCF to VHCF, at a given stress level, both surface and subsurface nucleation may operate independently and therefore compete, each with a different corresponding variability [25], [26]. The assumption of independence is a first order simplification that is particularly adequate during 
the crack incubation period, which comprises a significant portion of the overall life in the HCF and VHCF regimes. This incubation period comprises the initial formation and growth of the dominant fatigue crack within the first grain/phase. Furthermore, modeling the transition in failure mode is intrinsically more complex since it requires the adequate characterization of microstructure attributes for each mechanism (e.g., grain size on the surface as well as inclusion morphology).

The characterization of fatigue life variability requires explicit consideration of microstructures in addition to a failure criteria or driving force that represents each active damage mechanism. In this sense, multiple driving forces or FIPs should be compared simultaneously to determine which mechanism yields the lowest fatigue life for a specific microstructure arrangement. The quality of the microstructure characterization and digital representation can affect the quantification of fatigue driving forces in various ways. For example, the assessment of the driving force for transgranular fatigue crack along a PSB based on the cyclic shear of slip planes is quite insensitive to the roughness of the grain boundary representation [9]. Indeed, plastic deformation in the bulk of the grain is usually very homogeneous along the slip band and depends more strongly on the relative stiffness of the neighbor grain.

On the contrary, the assessment of crack growth from a triple point junction or a partially debonded inclusion requires the quantification of the stress or strain gradients near the inhomogeneity and should convey a more detailed representation of the microstructure. Therefore, relatively coarse meshes can be adequate to assess transgranular failure in HCF scenarios, while conformal meshes that represent more accurately the details of microstructure attributes would be more appropriate to assess intergranular failure in the VHCF regime. In any case, there is no benefit to further element mesh refinement within grains below the intrinsic slip localization length scale, e.g., PSB or cell size, unless the structures are modeled explicitly. This length scale, typically on the order of one micron, depends on the temperature and serves as a practical lower limit for element size. Dislocation substructures are intrinsic material mechanisms that regularize singularities and mitigate the intensity of stress and strain gradients.

In addition to appropriate mesh refinement, a large number of grain clusters should be assessed. Most computational models are not large enough to constitute representative volume elements (RVEs), but rather consider smaller statistical volume elements (SVEs) comprising samples of microstructure. However, the definition of such volumes depends on the crack length range of interest (e.g., maximum allowed crack length) or the local plastic heterogeneity. In this sense, the level of plastic heterogeneity increases from LCF to VHCF, which suggests that increasingly larger number of grains within an RVE and number of SVE realizations are required for more accurate VHCF model prediction.

As a surface or subsurface crack forms and propagates away from the stress concentration at its origin, the driving force for crack extension initially decreases. Once this crack approaches a grain boundary, it can slow down or potentially arrest. Indeed, in both HCF and VHCF, mesoscale cracks may experience a delay of their rate of growth at a microstructure barrier until the crack front samples enough grains to build up a sufficient crack size and associated driving force to intermittently overcome the 
microstructure barriers, eventually transitioning to an apparent smooth growth character. After this condition has been achieved, the similitude hypothesis can be assumed and singularity based fracture mechanics theories are valid. Before this, FIPs that account for the effect of microstructure on fatigue driving force and barriers inhibiting crack growth should be considered as a means to assess the dominant failure mode [27], [28].

\section{Mesoscale driving forces for HCF and VHCF}

Traditional fracture mechanics approaches rely on macroscopic fatigue driving force quantities such as $\Delta \mathrm{K}$ or $\Delta \mathrm{J}$ to estimate fatigue crack growth. These quantities invoke singularity formulations that do not explicitly consider the nature of distributed crystallographic slip, microstructure attributes, nor distinct failure mode or mechanisms. Even micromechanical forms of $\Delta K$ that seek to reflect the local driving force, though commonly used, are limited to small scale yielding and other tenets of LEFM. Mesoscale driving forces are formulated with regard to specific failure mechanisms operating over physically-meaningful domains comprising the damage process zone (DPZ), as shown in Figure 1.

For transgranular failure, much research has shown that the inelastic shear strain range correlates with fatigue damage. As shown by Hochhalter et al. [29], it is difficult to determine which FIP descriptor is the best to quantify fatigue damage. The reason for this is two-fold: on the one hand, experimental characterization is usually limited to a 2-dimensional description at discrete snapshots, with significant uncertainty regarding the local elastic and plastic deformation. Indeed, 3-dimensional characterization of fatigue crack growth is rather difficult to obtain. Additionally, modeling approaches calibrated with macroscopic stress-strain data convey limited accuracy at the mesoscale. This is due to the fact that a focus on the response of polycrystalline aggregates can obscure local mechanisms and yet still follow the macroscopic cyclic stress-strain behavior.

Bennett and McDowell [30], [31] and McDowell [27] introduced the notion of a range of FIPs that reflect in more explicit manner the relative roles of reversed and cumulative directional slip at the scale of individual grains or crystalline regions in polycrystals (i.e., microplasticity). For example, the FatemiSocie [32], [33] shear-based FIP has been shown to correlate multiaxial fatigue crack initiation data very well in both LCF and HCF regimes at the grain scale and above [34], [28], [35]. It is defined by

$$
F I P_{F S}=\frac{\Delta \gamma_{\max }^{p^{*}}}{2}\left(1+K^{\prime} \frac{\sigma_{\max }^{n^{*}}}{\sigma_{y}}\right)
$$

where $\Delta \gamma_{\max }^{p^{*}} / 2$ is the nonlocal maximum cyclic plastic shear strain range averaged over a finite volume of material (typically on the order of several $\left.\mu \mathrm{m}^{3}\right), \sigma_{\max }^{n^{*}}$ and $\sigma_{y}$ are the maximum stress normal to the plane of $\Delta \gamma_{\max }^{p^{*}} / 2$ and cyclic yield strength, respectively, and constant $K^{\prime}$ mediates the influence of normal stress. Castelluccio and McDowell [36] later extended this FIP to a crystallographic form, i.e., 


$$
\operatorname{FIP}^{(\alpha)}=\left.\frac{1}{2} \Delta \gamma^{(\alpha)}\right|_{c y c}\left[1+k \frac{\sigma_{n}^{(\alpha)}}{\sigma_{y}}\right]
$$

where $\left.\Delta \gamma\right|^{(\alpha)}$ cyc is the cyclic plastic shear strain range on the $\alpha^{\text {th }}$ slip system and $\sigma_{n}^{(\alpha)}$ is the peak stress normal to the plane of the slip system. A similar but distinct parameter has been introduced by McDowell [27] to address correlations of directional plastic strain accumulation with crack formation due to grain or phase boundary impingement (Zener pileup [16], [37]), i.e.,

$$
F I P_{Z P}=\gamma_{n e t}^{p^{*}}\left(1+K_{z}^{\prime} \frac{\sigma_{G B}^{n^{*}}}{\sigma_{y}}\right)
$$

where $\gamma_{n e t}^{p^{*}}=\max \left(n_{i} \varepsilon_{i j}^{p^{*}} m_{j}\right)$ among all planes with unit tangent and normal vectors $\mathbf{m}$ and $\mathbf{n}$, respectively, is the maximum net plastic shear strain averaged over a finite volume of material, $\sigma_{G B}^{n^{*}}$ is the average peak stress normal to a boundary segment impinged by this slip, and $K_{z}^{\prime}$ is a constant that mediates the effect of this normal stress. Castelluccio and McDowell [38] revisited the intergranular FIP and proposed a formulation with two multiplicative terms, the first relating to the strength of dislocation pile-ups impinging on the GB and the second to the stress normal to the boundary, i.e.,

$$
\mathrm{FIP}_{\text {int }}=\left[\sum_{\alpha}\left|\frac{\gamma_{p}^{\alpha}}{2}\left(1+k_{\text {tran }} \frac{\sigma_{n}^{\alpha}}{\sigma_{y}}\right)\right|\right]^{\lambda_{1}}\left(\frac{\sigma_{n}^{G B}}{\sigma_{y}}\right)^{\lambda_{2}},
$$

where $\lambda_{1}$ and $\lambda_{2}$ are material constants, $\gamma_{p}^{\alpha}$ is the net directional/cumulative plastic shear for slip system $\alpha$, and $\sigma_{n}^{G B}$ is the peak stress normal to the grain boundary.

The multiple slip mechanism for elevated temperature microstructurally small crack growth in Nibased superalloys was modeled by Musinski and McDowell [39], [40]. To mimic the resultant plane of zigzag cross slip along discrete crystallographic planes, propagation along a "FIP-weighted" average crack plane was considered as

$$
\boldsymbol{n}_{\text {multi-slip }}=\frac{\sum_{m=1}^{N_{\text {sslip }}} F I P^{(\alpha)} \cdot \boldsymbol{n}^{(\alpha)}}{\left\|\sum_{m=1}^{N_{-} s l i p} F I P^{(\alpha)} \cdot \boldsymbol{n}^{(\alpha)}\right\|}
$$

where N_slip = 2 was used to model multiple slip along conjugate slip planes. This FIP was able to represent the failure mode and surface roughness of the fracture surface seen in experiments [39], [40]. 
Another commonly used FIP for fatigue crack nucleation [41] is based on the cumulative plastic strain defined by

$$
F I P_{\mathrm{p}}=\int_{0}^{\mathrm{t}} \sqrt{\dot{\varepsilon}_{\mathrm{ij}}^{\mathrm{p}} \dot{\varepsilon}_{\mathrm{ij}}^{\mathrm{p}}} \mathrm{d} \tau
$$

This kind of FIP has been correlated with the site of fatigue crack nucleation within individual surface grains [42], [43] for Ni-base superalloys. Motivated by the aforementioned model of Tanaka and Mura [44], Shenoy et al. [45] employed a FIP of the form

$$
F I P_{\mathrm{TM}}=\frac{\mathrm{d}_{\mathrm{g}}}{\alpha_{\mathrm{g}}}\left(\frac{\Delta \gamma_{\text {max }}^{\mathrm{p}^{*}}}{2}\right)^{2}
$$

for fatigue crack nucleation in Ni-base superalloy IN 100, where the dependence on grain size $d_{g}$ is employed to reflect the enhancement of localized slip with increase in the mean free path; the nucleation life is the inverse of this particular FIP, with $\alpha_{\mathrm{g}}$ as a fitting parameter.

Similar FIPs have been introduced to model driving forces for fatigue crack nucleation, including the proposition of Kirane and Ghosh [46], [47] of a generalized nonlocal cohesive relation based on coarsescale (microns) shear and opening tractions that appeals to buildup of elastic energy associated with dislocation pileups to drive crack nucleation. Indeed, a myriad of parameters have been proposed based on correlation with crack growth, but we emphasize that correlation does not imply causality and multiple FIPs might be valid under specific conditions. Furthermore, each FIP formulation is associated with a specific failure mechanism and can be applied to correlate fatigue crack formation in HCF or VHCF regimes.

Mesoscale driving forces at the scale of the fatigue DPZ as expressed by FIPs should be taken as approximate proxies or surrogates of the collective action of fundamental mechanisms operating at smaller length scales. The accuracy of these FIPs is improved when they are evaluated over a physically meaningful domain; this approach is a means to incorporate additional information such as observed crack paths or localization regions. For instance, in polycrystalline metallic alloys with grain sizes above one micron, transgranular fatigue crack formation occurs due to the cyclic irreversible deformation in the bulk of the grain. The crack growth rate could be precisely characterized by the mechanically irreversible crack tip emission of dislocations, which determine the change in crack tip opening and sliding displacements. Indeed, atomistic and dislocation dynamics simulations have been applied to model cyclic crack growth. However, these approaches are not powerful enough for engineering assessments at microstructural mesoscale comprising hundreds of grains and/or phases. Instead, continuum proxies must be employed.

Fatigue damage develops within a process zone rather than at a material point. For example, a persistent slip band forms across a grain cross section and the accumulation of irreversible deformation 
may occur at different points prior to adjoining the dominant crack [48-49]. Therefore, it is not sufficient to assess a local measurement of the cyclic irreversibility, but rather over a finite DPZ; in the context of discrete continuum units such as a finite element, this factor becomes even more important to mitigate mesh sensitivity. For transgranular HCF crack growth, the grain volume or the grain cross section oriented along slip planes (crystallographic bands, Figure 2, Left) offers a means to assess FIPs over a finite DPZ [50]. The assessment along bands is more refined and conveys a more relevant characterization of intrinsic material variability than FIP averages taken over entire grains, particularly at low applied strain amplitudes. Indeed, at higher strain amplitudes, plastic deformation becomes more homogeneous, with larger number of slip planes activated, and larger rotations. In the case of intergranular fatigue crack formation, damage accumulates at multiple process zones distributed along grain boundary sectors, which correspond to DPZs (Figure 2, Right).

In the transition between HCF and VHCF, plastic strain gradients within individual grains are more pronounced and crystallographic bands rather than the entire grain may more closely represent the DPZ. In the VHCF regime, the plastic deformation is strongly linked to the morphology of the microstructure feature that induces the stress concentration (e.g., strong stress or strain gradients from inclusions, voids, etc). In these cases, the driving force should be evaluated in terms of the local gradient of plastic ranges (e.g., the domain with the highest driving force) and updated according to the crack path. For example, the driving force of a partially debonded inclusion can be assessed by computing FIPs along the vicinity of the debonded perimeter [8]. The analysis of this perimeter smoothes out local variability, yielding values amenable for comparison with experimental data such as beach marks. Furthermore, crack extension may be implemented in discrete increments of the entire perimeter, which reduces the computational burden and updates the driving force a limited number of times (affecting the degree of accuracy) [51].

Salajegheh and McDowell [8] have studied the problem of probability of surface versus subsurface fatigue crack initiation at large grains and/or inclusions using the FIP approach. They assumed that the number of surface fatigue critical hotspots followed a binomial distribution and bulk fatigue hotspots were critical only when surface hotspots were absent. Using this framework, they showed trends of increasing probability of surface crack formation and early growth with increasing specimen size, increasing inclusion number density, increasing inclusion size distribution, and decreasing inclusion/matrix interface strength. Such a probabilistic framework is useful in the transition from HCF (surface-dominated) to VHCF (bulkdominated) regimes.

A final note pertains to ultra-fine grain materials with grain sizes below one micron (i.e., interfacemediated response). Plastic deformation of these materials is strongly influenced by the relatively large ratio of grain surface area to volume. Indeed, dislocation nucleation in the bulk of a grain tends to be suppressed in favor of dislocations emitted from the interface. These mechanisms differ from those in most engineering metallic materials. For instance, recent investigations have highlighted the role of vacancies in forming surface slip bands and subsurface voids that eventually connect and extend to form fatigue cracks [13], [14]. These mechanisms should be captured by new appropriate FIP formulations. 


\section{Dominant hierarchy in HCF and VHCF regimes}

Sequential processes of fatigue crack formation and early growth within the first few grains or phases of a polycrystalline metal typically follow a hierarchy of stages and corresponding length scale regimes. Figure 3 presents a decomposition of the multiple stages of crack formation and growth, where $\mathrm{N}_{\text {nucl }}$ pertains to crack nucleation within the first grain or phase, and $\mathrm{N}_{\mathrm{MSC}}, \mathrm{N}_{\mathrm{PSC}}$ and $\mathrm{N}_{\mathrm{LC}}$ are the number of cycles needed to grow microstructurally small, physically small, and mechanically long cracks; the latter meets similitude requirements of fracture mechanics. It is now well established that longer fatigue lives in the HCF regime and beyond are usually due to a delay in the nucleation and microstructurally small crack growth stages. Therefore, most efforts to estimate fatigue lives in the HCF and VHCF regimes attempt to characterize and predict $\mathrm{N}_{\text {nucl }}$ and $\mathrm{N}_{\mathrm{MSC}}$. Both stages should be considered since a large fraction of the fatigue variability is explained by the meandering nature of fatigue cracks in the microstructurally small crack growth stage [36]. The behavior in the VHCF regime depends critically on the nucleation and growth across the first few strong microstructural barriers.

The hierarchical multistage approach to fatigue crack formation and early growth in Figure 3 has been laid out in reviews by McDowell [27] and McDowell and Dunne [41]. The fatigue crack driving forces at each stage can be quantified with surrogate FIPs, as shown by McDowell and colleagues for microstructure-sensitive polycrystal plasticity models for duplex Ti-6Al-4V [53]-[55], $\gamma-\gamma^{\prime}$ Ni-base superalloys [36], [56], [45], [57], and martensitic steels [58]-[60]. In the context of polycrystal plasticity, FIPs quantify the relative potency to nucleate and grow cracks at the mesoscale by assigning a relation between the number of cycles necessary to nucleate or grow a crack at the scale of the nonlocal averaging volume and the associated FIP, i.e., $\mathrm{N}_{\text {nucl }}=f(\mathrm{FIP}),\left.\frac{\mathrm{da}}{\mathrm{dN}}\right|_{\mathrm{msc}}=\mathfrak{J}^{*}(\mathrm{FIP})$. Furthermore, FIPs and associated life relations can appeal to some form of continuously distributed dislocation theory (cf. Ref [44]) or some variant of a Griffith energy balance that loosely incorporates various terms associated with energy release and transfer to dislocation plasticity and new crack surfaces (cf. Refs [44], [61], [62]). Such formulations typically "smear" much of the detail of the underlying dislocation fields.

McDowell and co-workers [36], [45] have also applied a MSC regime growth law for Stage I crystallographic growth that employs a two parameter FIP of the form appearing in Eqs. (1) and (2) (sliding controlled by cyclic plastic shear strain, with an additional effect of peak normal stress to this plane), where the analogy to mixed mode $\Delta J$-integral [63] and cyclic crack tip displacement range $\Delta C T D$ [64] is made, i.e.,

$$
\left.\frac{\mathrm{da}}{\mathrm{dN}}\right|_{\mathrm{msc}}=\mathfrak{I}(\mathrm{FIP})-\Delta \mathrm{CTD}_{\mathrm{th}}=\phi \Delta \mathrm{CTD}-\eta \mathrm{b}
$$


Here, $\phi$ is an irreversibility scaling factor, $\Delta \mathrm{CTD}_{\text {th }}$ is a threshold crack tip displacement range below which the crack will not grow, $n \approx 1$ and $b$ is the magnitude of the Burgers vector. Clearly, relation (8) employs the coarse scale FIP as a surrogate driving force parameter that is intended to parameterize the dependence of the fine scale propagation relation on $\triangle \mathrm{CTD}\left(\Delta \mathrm{CTD}=\sqrt{\Delta \mathrm{CTSD}^{2}+\Delta \mathrm{CTOD}^{2}}\right)$. In Eq. (8), an equivalence of the coarse scale relation is implied to a fine scale relation in the crack tip process zone via the second equality, where $\phi$ is an irreversibility factor that controls cyclic crack advance associated with the fraction of process zone dislocations that do not return to the crack tip during unloading [65].

The understanding of thresholds in constitutive models and in crack growth models is essential when predicting the activation or suppression of given failure mechanisms in a given fatigue regime. For example, Musinski and McDowell [66], [67] simulated strain-life curves using a hierarchical multistage fatigue life approach for the prediction of life in the LCF to VHCF regimes with and without inclusions. For IN100 Ni-base superalloy, the microstructure-sensitive constitutive model employed [66] contained a two term flow rule, the first term accounting for plasticity above a threshold slip resistance and the second term accounting for thermally-activated creep at lower stresses. A fit of the fatigue crack nucleation and growth of the model in the LCF was shown to be sufficient to predict the strain-life slope in the HCF regime (cf. Figure 4). Accordingly, it is understood that the conventional Basquin's Law in HCF is a natural consequence of heterogeneity of cyclic plastic deformation among grains (microplasticity) rather than a fundamental scaling relation in its own right. A subsequent drop off of the curve in the VHCF regime corresponded to the absence of first term flow rule mediated microplasticity and the predominance of lower stress thermally-activated creep plasticity (second term) in the flow rule. In addition to microplasticity within grains, cracks can forms at inclusions located in the bulk of the material. Indeed, differences in failure mechanisms that are dominated by grain-to-grain interactions versus defect-driven (e.g. inclusion) augmentation often compete in the transition from HCF to VHCF regimes. As failure mechanisms complete at lower applied strain amplitudes, the size of a given flaw relative to average grain size (for example, Fig. 4), crack initiation size, and distance of the flaw to the surface can become important. The size of the nucleated crack can have a significant effect on whether it will continue to grow past the first few microstructural barriers.

6. Challenges for microstructure-sensitive modeling in the HCF and VHCF regimes

One of the main challenges in bridging fatigue models and experiments is innovating with techniques that provide an efficient practical means to compare fatigue resistance of various microstructures, thereby avoiding the need for hundreds of costly and time consuming experiments [68]. Such models should also have tractable computational requirements. Indeed, conventional loading frequencies (up to about $100 \mathrm{~Hz}$ ) would take years to complete a single test in the VHCF regime. Therefore, ultrasonic fatigue testing, which makes use of the natural frequency of the specimen being tested [2], has 
been developed to reduce the time required to run a VHCF test. When incorporating these types of methods, experimentalists must carefully control energy dissipation and applied stresses, but must also verify that the failure mechanisms during ultrasonic fatigue testing are consistent with the failure mechanisms present in tests at frequencies and strain amplitudes of the actual application.

One experimental approach that facilitates the observation of fatigue damage has been the use of sharp notches to define the location of initiation of a fatigue crack. Focused ion beam (FIB) cutting can produce notches that impose a "crack initiator" at the surface in the VHCF regime. For example, Geathers et al. [69], have combined an ultrasonic fatigue specimen and an SEM to study the effect of environment on small crack growth in Ti-6242S at very low applied stresses. This approach can effectively characterize the microstructure variability of crack growth rates, but cannot assess the fatigue nucleation variability, which accounts for a significant fraction of the total variability in the VHCF regime. Similarly, several approaches have attempted to model crack growth from notches in the HCF-VHCF by assessing the driving force intensity at the notches [48]. Although promising, these techniques should consider the effect of crack arrest during crack growth.

Given the time demands of the experimental characterization of fatigue damage variability in the VHCF regime, multiscale modeling can become an effective tool to identify microstructure attributes that control fatigue life and give rise to variability. However, modeling approaches have a major challenge in predicting accurate stress and strain fields within grains and in characterizing the spatial and size distribution of various competing microstructure attributes. The detailed characterization of constitutive models at the mesoscale is a priority to improve the prediction of fatigue lives. For example, MüllerBollenhagen et al. [5] showed that the content of martensite formation has a non-monotonic impact on VHCF fatigue lives in austenitic stainless steels. Thus, the volume content of martensite needs to be accurately predicted under various loading conditions and histories.

In addition to use of more realistic microstructures [70], constitutive models employed to model VHCF should be validated by considering stresses and strains at the microscale, not just a macroscale stress-strain curve. This can be pursued for example by in situ mapping of strains using digital image correlation as a means to experimentally probe grain-level strains within a given microstructure albeit limited to surface measurements. Imaging via synchrotron radiation is another route. Another strategy is the calibration based on responses of single crystals, additionally accounting for grain size effects. In addition, modeling strategies should also consider (implicitly or explicitly) time separation schemes to approximate the condition of materials after millions of loading cycles without the need for detailed tracking of the evolution on a cycle-by-cycle basis. These remain as substantial challenges.

Another issue to consider in nucleation and early growth of cracks formed at subsurface inclusions in high strength bearing steels under VHCF conditions, for example, is the observation of a so-called fine granular area (FGA) around the inclusion which controls the great majority of lifetime for subsurface inclusion-FGA-fisheye induced failures, as reviewed by Li et al. [71], and reported in some prior works by 
Sakai and colleagues [72-73]. Prasannavenkatesan et al. [74] have discussed the challenges in modeling transient cyclic deformation and microstructure refinement that can occur over many cycles in fully martensitic gear steels. Microstructure surrounding cracked or debonded inclusions may evolve and refine with the progression of localized cyclic plastic deformation and microstructurally small crack growth, potentially affected as well by environmental effects if near the surface. Related phenomena are critically important in the VHCF regime and are extremely challenging to fully understand since the processes are subsurface and not readily accessible via in situ measurement. As a result, the precise cause and effect of physical processes, including the overall partitioning of crack formation and early growth processes within the affected microstructure, remain as open issues.

One of the main challenges in integrating computational and experimental microstructure-level data is a means to track the process flow of data and compare/contrast the 3D metadata from each experiment or simulation. Data science can provide a useful assistance in designing a network to compare, correlate and contrast large 3D datasets. This type of data can be used to detect subtle differences in microstructure features and whether there are any sensitivities to them. For example, one technique that has received great traction is the building of 3D actual or synthetic microstructures, respectively, from serial sectioning experiments or statistical aspects of microstructure features using the DREAM.3D software [70], for example. With this tool, multiple digital synthetic microstructures can be created to assess the effect of aleatory microstructure uncertainty on overall fatigue variability.

Finally, there is a clear need for shifting our interpretation of experimental and computational results in the HCF to VHCF regimes via probabilistic approaches (e.g., Refs. [8], [26]) rather than traditional deterministic approaches common in LCF. Competing failure modes and transition from surface to subsurface crack formation can and should be understood in a probabilistic context.

\section{Conclusions}

Fatigue failure in the HCF and VHCF regimes is the result of irreversible mechanisms at hot spots that serve as crack embryos. The chances that these embryos form a fatigue crack depends on factors that are not unique and vary with the intensity of the loading, the environment, microstructure details, etc. Multiple fatigue damage mechanisms can operate simultaneously. Surface failure is most typical in HCF due to the enhancement of the environment on irreversible deformation. Subsurface damage may occur even if it might not lead to failure. Under certain circumstances, different failure mechanisms can operate independently through most of the fatigue lives, which yields statistically independent fatigue life ensembles. In this case, the experimental and modeling characterization can be performed assuming that just one mechanism dominates fatigue failure. Modeling each failure mechanism requires a measure of the fatigue driving force and a volume associated with the damage process zone. Surface failure is usually related to the cyclic strains along slip bands across grains or impinging on grain boundaries. On the contrary, subsurface failure depends strongly on the morphology of the microstructure feature (intrinsic 
or extrinsic) that induces a local stress concentration. These differences should be accounted for in the quantification of the driving forces when modeling the transition from HCF to VHCF.

Further advance in experiments and models should convey more hierarchical fidelity in both the description of the microstructure morphology and the stress/strain fields that drive fatigue crack formation and early growth through the microstructure. Moreover, constitutive models for cyclic inelastic deformation must reflect local slip conditions and not just those of the average polycrystal. We noted that most constitutive models will not accurately predict the partition of strains among slip systems next to an inclusion, which can be mitigated by assessing non-local volumes associated with specific failure modes. The size of a fatigue damage process zone depends on the specific mechanism activated, so fatigue damage should be assessed over domains that correlate with physical reasoning and experimental evidence. In addition, each mechanism has independent requirements for mesh refinement and degree of accuracy in representation of microstructure morphology.

We conclude by highlighting that as computational power continues to increase, higher fidelity approaches will begin to play an increasing role in assessing fatigue phenomena. Nevertheless, the limitation of such approaches assures the continued need for experimental work to determine which mechanisms dominate fatigue damage. Both multiscale experiments that build on the foundations laid by Claude Bathias [1-2] and higher fidelity models such as atomistic, phase field, and dislocation dynamics are necessary to successfully develop mesoscale models that correlate fatigue damage in the HCF and VHCF regimes.

\section{Acknowledgements}

DLM is grateful for the support of NSF CMMI-1333083 on Microstructure-Sensitive Design of Multiphase Structural Alloys, as well as the Carter N. Paden, Jr. Distinguished Chair in Metals Processing at Georgia Tech. 


\section{$\underline{\text { References }}$}

[1] C. Bathias, "There is no infinite fatigue life in metallic materials," Fatigue Fract. Eng. Mater. Struct., vol. 22, no. 7, pp. 559-565, 1999.

[2] C. Bathias and P. C. Paris, Gigacycle Fatigue in Mechanical Practice, Marcel Dekker, New York, 2005.

[3] K. Miller, "The Three Thresholds for Fatigue Crack Propagation," in Fatigue and Fracture Mechanics: 27th volume, R. S. Piascik and J. C. Newman, Eds. ASTM, 1997, pp. 267-286.

[4] S. E. Stanzl-Tschegg and B. Schönbauer, "Mechanisms of strain localization, crack initiation and fracture of polycrystalline copper in the VHCF regime," Int. J. Fatigue, vol. 32, no. 6, pp. 886-893, Jun. 2010.

[5] C. Müller-Bollenhagen, M. Zimmermann, and H.-J. Christ, "Very high cycle fatigue behaviour of austenitic stainless steel and the effect of strain-induced martensite," Int. J. Fatigue, vol. 32, no. 6, pp. 936-942, Jun. 2010.

[6] H. Mughrabi, "On 'multi-stage' fatigue life diagrams and the relevant life-controlling mechanisms in ultrahigh-cycle fatigue," Fatigue Fract. Eng. Mater. Struct., vol. 25, no. 8-9, pp. 755-764, 2002.

[7] J. Petit and C. Sarrazin-Baudoux, "An overview on the influence of the atmosphere environment on ultra-high-cycle fatigue and ultra-slow fatigue crack propagation," Int. J. Fatigue, vol. 28, no. 11, pp. 1471-1478, Nov. 2006.

[8] N. Salajegheh and D. L. McDowell, "Microstructure-sensitive weighted probability approach for modeling surface to bulk transition of high cycle fatigue failures dominated by primary inclusions," Int. J. Fatigue, vol. 59, pp. 188-199, 2014.

[9] G. M. Castelluccio and D. L. McDowell, "Microstructure and mesh sensitivities of mesoscale surrogate driving force measures for transgranular fatigue cracks in polycrystals," Mater. Sci. Eng. A, vol. 639, pp. 626-639, Jul. 2015.

[10] J. Polak, K. Obrtlik, M. Hajek, and A. Vasek, "Cyclic stress-strain response of polycrystalline copper in a wide range of plastic strain amplitudes," Mater. Sci. Eng. A, vol. 151, no. 1, pp. 19-27, 1992.

[11] H. Matsunaga and H. Noda, "Visualization of Hydrogen Diffusion in a Hydrogen-Enhanced Fatigue Crack Growth in Type 304 Stainless Steel," Metall. Mater. Trans. A, vol. 42, no. 9, pp. 2696-2705, Sep. 2011.

[12] I. Aubert, F. Plessier, N. Saintier, and J. Olive, "Modification of Plastic Strain Localization Induced by Hydrogen Absorption," Adv. Mater. Sci., vol. 8, no. 1, pp. 5-14, Mar. 2008.

[13] P. Lukáš, L. Kunz, L. Navrátilová, and O. Bokůvka, "Fatigue damage of ultrafine-grain copper in veryhigh cycle fatigue region," Mater. Sci. Eng. A, vol. 528, no. 22-23, pp. 7036-7040, Aug. 2011.

[14] L. Kunz, P. Lukáš, and L. Navrátilová, "Strain localization and fatigue crack initiation in ultrafinegrained copper in high- and giga-cycle region," Int. J. Fatigue, vol. 58, pp. 202-208, Jan. 2014.

[15] H. Mughrabi and H. W. Hoppel, "Cyclic deformation and fatigue properties of very fine-grained metals and alloys," Int. J. Fatigue, vol. 32, no. 9, pp. 1413-1427, Sep. 2010.

[16] A. N. Stroh, "The Formation of Cracks as a Result of Plastic Flow," Proc. R. Soc. Lond. Ser. A, vol. 223, no. 1154, pp. 404-414, 1954.

[17] S. Suresh, Fatigue of materials, 2nd ed. Cambridge University Press, 1998.

[18] A. Weidner, D. Amberger, F. Pyczak, B. Schönbauer, S. Stanzl-Tschegg, and H. Mughrabi, "Fatigue damage in copper polycrystals subjected to ultrahigh-cycle fatigue below the PSB threshold," Int. J. Fatigue, vol. 32, no. 6, pp. 872-878, Jun. 2010.

[19] C. Stocker, M. Zimmermann, and H.-J. Christ, "Localized cyclic deformation and corresponding dislocation arrangements of polycrystalline Ni-base superalloys and pure Nickel in the VHCF regime," Int. J. Fatigue, vol. 33, no. 1, pp. 2-9, 2011. 
[20] T. Sakai, N. Oguma, and A. Morikawa, "Microscopic and nanoscopic observations of metallurgical structures around inclusions at interior crack initiation site for a bearing steel in very high-cycle fatigue," Fatigue Fract. Eng. Mater. Struct., vol. 38, no. 11, pp. 1305-1314, Nov. 2015.

[21] R. J. Morrissey and P. J. Golden, "Fatigue strength of a single crystal in the gigacycle regime," Int. J. Fatigue, vol. 29, no. 9-11, pp. 2079-2084, Sep. 2007.

[22] Y. Murakami, T. Nomoto, T. Ueda, and Y. Murakami, "On the mechanism of fatigue failure in the superlong life regime ( $\mathrm{N}>107$ cycles). Part 1: influence of hydrogen trapped by inclusions," Fatigue Fract. Eng. Mater. Struct., vol. 23, no. 11, pp. 893-902, Nov. 2000.

[23] Y. Murakami, T. Nomoto, T. Ueda, and Y. Murakami, "On the mechanism of fatigue failure in the superlong life regime ( $\mathrm{N}>107$ cycles). Part II: influence of hydrogen trapped by inclusions," Fatigue Fract. Eng. Mater. Struct., vol. 23, no. 11, pp. 903-910, Nov. 2000.

[24] G. T. Cashman, "A review of competing modes fatigue behavior," Int. J. Fatigue, vol. 32, pp. 492496, 2010.

[25] K. S. Ravi Chandran, P. Chang, and G. T. Cashman, "Competing failure modes and complex S-N curves in fatigue of structural materials," Int. J. Fatigue, vol. 32, no. 3, pp. 482-491, Mar. 2010.

[26] S. K. Jha, M. J. Caton, and J. M. Larsen, "A new paradigm of fatigue variability behavior and implications for life prediction," Mater. Sci. Eng. -Struct. Mater. Prop. Microstruct. Process., vol. 468, pp. 23-32, 2007.

[27] D. L. McDowell, "Simulation-based strategies for microstructure-sensitive fatigue modeling," Mater. Sci. Eng. A, vol. 468, pp. 4-14, 2007.

[28] D. L. McDowell, "Mutiaxial Fatigue Strength," in ASM handbook on Fatigue and Fracture, vol. 19, Metals Park, OH: American society of metals, 1996, pp. 263-273.

[29] J. D. Hochhalter, D. J. Littlewood, M. G. Veilleux, J. E. Bozek, A. M. Maniatty, A. D. Rollett, and A. R. Ingraffea, "A geometric approach to modeling microstructurally small fatigue crack formation: III. Development of a semi-empirical model for nucleation," Model. Simul. Mater. Sci. Eng., vol. 19, no. 3, p. 035008, 2011.

[30] V. P. Bennett and D. L. McDowell, "Cyclic crystal plasticity analyses of stationary, microstructurally small surface cracks in ductile single phase polycrystals," Fatigue Fract. Eng. Mater. Struct., vol. 25, no. 7, pp. 677-693, 2002.

[31] V. P. Bennett and D. L. McDowell, "Polycrystal orientation distribution effects on microslip in high cycle fatigue," Int. J. Fatigue, vol. 25, no. 1, pp. 27-39, Jan. 2003.

[32] A. Fatemi and D. F. Socie, "A critical plane approach to multiaxial fatigue damage including out-ofphase loading," Fatigue Fract. Eng. Mater. Struct., vol. 11, no. 3, pp. 149-165, 1988.

[33] A. Fatemi and P. Kurath, "Multiaxial Fatigue Life Predictions Under the Influence of mean-stresses," J. Eng. Mater. Technol., vol. 110, no. 4, pp. 380-388, 1988.

[34] D. F. Socie, "Critical Plane Approaches for Multiaxial Fatigue Damage Assessment," in Advances in Multiaxial Fatigue, ASTM, 1993, pp. 7-36.

[35] H. Vehoff, A. Nykyforchyn, and R. Metz, "Fatigue crack nucleation at interfaces," Mater. Sci. Eng. A, vol. 387-389, pp. 546-551, Dec. 2004.

[36] G. M. Castelluccio and D. L. McDowell, "A mesoscale approach for growth of 3D microstructurally small fatigue cracks in polycrystals," Int. J. Damage Mech., vol. 23, no. 6, p. 1056789513513916, 2014.

[37] H. Fan, "Interfacial Zener-Stroh Crack," J. Appl. Mech., vol. 61, no. 4, pp. 829-834, 1994.

[38] G. M. Castelluccio and D. L. McDowell, "Mesoscale modeling of microstructurally small fatigue cracks in metallic polycrystals," Mater. Sci. Eng. A, vol. 598, pp. 34-55, Mar. 2014.

[39] W. D. Musinski and D. L. McDowell, "Simulating the effect of grain boundaries on microstructurally small fatigue crack growth from a focused ion beam notch through a three-dimensional array of grains," Acta Mater., vol. 112, pp. 20-39, 2016. 
[40] W. D. Musinski, "Modeling the effects of shot-peened residual stresses and inclusions on microstructure-sensitive fatigue of Ni-base superalloy components," Ph.D. Thesis, Georgia Institute of Technology, 2014.

[41] D. L. McDowell and F. P. E. Dunne, "Microstructure-sensitive computational modeling of fatigue crack formation," Int. J. Fatigue, vol. 32, no. 9, pp. 1521-1542, 2010.

[42] A. Manonukul and F. P. E. Dunne, "High- and low-cycle fatigue crack initiation using polycrystal plasticity," Proc. R. Soc. Lond. Ser. A, vol. 460, no. 2047, pp. 1881-1903, 2004.

[43] F. P. E. Dunne, A. J. Wilkinson, and R. Allen, "Experimental and computational studies of low cycle fatigue crack nucleation in a polycrystal," Int. J. Plast., vol. 23, no. 2, pp. 273-295, 2007.

[44] K. Tanaka and T. Mura, "A Dislocation Model for Fatigue Crack Initiation," J. Appl. Mech., vol. 48, no. 1, pp. 97-103, 1981.

[45] M. M. Shenoy, J. Zhang, and D. L. McDowell, "Estimating fatigue sensitivity to polycrystalline Ni-base superalloy microstructures using a computational approach," Fatigue Fract. Eng. Mater. Struct., vol. 30, no. 10, pp. 889-904, 2007.

[46] K. Kirane and S. Ghosh, "A cold dwell fatigue crack nucleation criterion for polycrystalline Ti-6242 using grain-level crystal plasticity FE Model," Int. J. Fatigue, vol. 30, no. 12, pp. 2127-2139, 2008.

[47] K. Kirane, S. Ghosh, M. Groeber, and A. Bhattacharjee, "Grain Level Dwell Fatigue Crack Nucleation Model for Ti Alloys Using Crystal Plasticity Finite Element Analysis," J. Eng. Mater. Technol., vol. 131, no. 2, p. 021003 1-14, 2009.

[48] E. Ferrie and M. Sauzay, "Influence of Local Crystallographic Orientation on Short Crack Propagation in High Cycle Fatigue of 316LN Steel," J Nucl Mater, Vol. 386-388, pp. 666-9, 2009.

[49] J. Polak and M. Sauzay, "Growth of Extrusions in Localized Cyclic Plastic Straining," Mater Sci Eng A, Vol. 500, pp.122-129, 2009.

[50] G. M. Castelluccio, W. D. Musinski, and D. L. McDowell, "Recent developments in assessing microstructure-sensitive early stage fatigue of polycrystals," Curr. Opin. Solid State Mater. Sci., vol. 18, no. 4, pp. 180-187, 2014.

[51] B. C. Clark, G. M. Castelluccio, M. Reiterer, R. W. Neu, and D. L. McDowell, "Structure-property Tools for Fatigue Performance of MP35N Medical-Grade Wire," presented at the ASME Applied Mechanics and Materials Conference, Seattle, Washington, USA, 2015.

[52] B. Kunkler, O. Duber, P. Koster, U. Krupp, C. P. Fritzen, and H. J. Christ, "Modelling of short crack propagation - Transition from stage I to stage II," Eng. Fract. Mech., vol. 75, no. 3-4, pp. 715-725, 2008.

[53] J. R. Mayeur and D. L. McDowell, "A three-dimensional crystal plasticity model for duplex Ti-6Al-4V," Int. J. Plast., vol. 23, no. 9, pp. 1457-1485, 2007.

[54] M. Zhang, J. Zhang, and D. L. McDowell, "Microstructure-based crystal plasticity modeling of cyclic deformation of Ti-6Al-4V," Int. J. Plast., vol. 23, no. 8, pp. 1328-1348, Aug. 2007.

[55] F. Bridier, D. L. McDowell, P. Villechaise, and J. Mendez, "Crystal plasticity modeling of slip activity in Ti-6Al-4V under high cycle fatigue loading," Int. J. Plast., vol. 25, no. 6, pp. 1066-1082, Jun. 2009.

[56] R. S. Kumar, A.-J. Wang, and D. L. Mcdowell, "Effects of Microstructure Variability on Intrinsic Fatigue Resistance of Nickel-base Superalloys - A Computational Micromechanics Approach," Int. J. Fract., vol. 137, no. 1-4, pp. 173-210, Jan. 2006.

[57] M. M. Shenoy, Y. Tjiptowidjojo, and D. L. McDowell, "Microstructure-sensitive modeling of polycrystalline IN100," Int. J. Plast., vol. 24, no. 10, pp. 1694-1730, 2008.

[58] J. Zhang, R. Prasannavenkatesan, M. M. Shenoy, and D. L. McDowell, "Modeling fatigue crack nucleation at primary inclusions in carburized and shot-peened martensitic steel," Eng. Fract. Mech., vol. 76, no. 3, pp. 315-334, 2009. 
[59] R. Prasannavenkatesan, J. Zhang, D. L. McDowell, G. B. Olson, and H.-J. Jou, "3D modeling of subsurface fatigue crack nucleation potency of primary inclusions in heat treated and shot peened martensitic gear steels," Int. J. Fatigue, vol. 31, no. 7, pp. 1176-1189, Jul. 2009.

[60] R. Prasannavenkatesan and D. L. McDowell, "Polycrystal Plasticity Modeling of Cyclic Residual Stress Relaxation in Shot Peened Martensitic Gear Steel," J. Eng. Mater. Technol., vol. 132, no. 3, pp. 031011-031011, Jun. 2010.

[61] G. Venkataraman, Y. W. Chung, and T. Mura, "Application of minimum energy formalism in a multiple slip band model for fatigue - I. Calculation of slip band spacings," Acta Metall. Mater., vol. 39, no. 11, pp. 2621-2629, Nov. 1991.

[62] G. Venkataraman, Y. W. Chung, and T. Mura, "Application of minimum energy formalism in a multiple slip band model for fatigue-II. Crack nucleation and derivation of a generalised CoffinManson law," Acta Metall. Mater., vol. 39, no. 11, pp. 2631-2638, Nov. 1991.

[63] T. Hoshide and D. F. Socie, "Mechanics of mixed mode small fatigue crack growth," Eng. Fract. Mech., vol. 26, no. 6, pp. 841-850, 1987.

[64] D. L. McDowell and J. Y. Berard, "A $\Delta \mathrm{J}$-based approach to Biaxial Fatigue," Fatigue Fract. Eng. Mater. Struct., vol. 15, no. 8, pp. 719-741, 1992.

[65] F. O. Riemelmoser, P. Gumbsch, and R. Pippan, "Dislocation modelling of fatigue cracks: An overview," Mater. Trans. Jim, vol. 42, no. 1, pp. 2-13, 2001.

[66] W. D. Musinski and D. L. McDowell, "Microstructure-sensitive probabilistic modeling of HCF crack initiation and early crack growth in Ni-base superalloy IN100 notched components," Int. J. Fatigue, vol. 37, pp. 41-53, 2012.

[67] W. D. Musinski and D. L. McDowell, "Microstructure-Sensitive Probabilistic Fatigue of Ni-Base Superalloy IN100: Modeling the effect of inclusions on strain-life curve in the HCF and VHCF regimes," in VHCF-5, Berlin, Germany, 2011, pp. 231-236.

[68] C. P. Przybyla and D. L. McDowell, "Microstructure-sensitive extreme value probabilities for high cycle fatigue of Ni-base superalloy IN100," Int. J. Plast., vol. 26, no. 3, pp. 372-394, 2010.

[69] J. Geathers, C. J. Torbet, J. W. Jones, and S. Daly, "Investigating environmental effects on small fatigue crack growth in Ti-6242S using combined ultrasonic fatigue and scanning electron microscopy," Int. J. Fatigue, vol. 70, pp. 154-162, 2015.

[70] M. Groeber and M. Jackson, "DREAM.3D: A digital representation environment for the analysis of microstructure in 3D," Integ. Mater. Manuf. Innov., vol. 3:5, pp. 1-17, 2014.

[71] W. Li, H. Deng, Z. Sun, Z. Zhang, L. Lu and T. Sakai, "Subsurface inclusion-induced crack nucleation and growth behaviors of high strength steels under very high cycle fatigue: Characterization and microstructure-based modeling," Mater. Sci. Eng. A, vol. 641, pp. 10-20, 2015.

[72] T. Sakai, N. Oguma and A. Morikawa, "Microscopic and nanoscopic observations of metallurgical structures around inclusions at interior crack initiation site for a bearing steel in very high-cycle fatigue," Fatigue and Fracture of Engineering Materials and Structures, vol. 38 (11), pp. 1305-11314, 2015.

[73] T. Sakai, Y. Sato and N. Oguma, "Characteristic S-N properties of high-carbon-chromium-bearing steel under axial loading in long-life fatigue," Fatigue and Fracture of Engineering Materials and Structures, vol. 24 (8-9), pp. 765-773, 2012.

[74] R. Prasannavenkatesan, C.P. Przybyla, N. Salajegheh and D.L. McDowell, "Simulated extreme value fatigue sensitivity to inclusions and pores in martensitic gear steels," Eng. Fract. Mech., vol. 78, pp. 1140-1155, 2011. 


\section{Figure Captions}

Figure 1: A damage process zone (DPZ) is defined the mesoscale and incorporates spatial information to estimate the driving force for fatigue crack formation and early growth. This strategy aims at mitigating the uncertainty associated with collective mesoscale processes involving dislocation substructures.

Figure 2: Schematic representation of DPZs. (Left) Crystallographic bands within which FIPs are averaged to estimate transgranular fatigue crack growth. (Right) 2D cross section of a mesh with numbered grains and some of the grain boundary sectors highlighted with intensified shade of color; intergranular FIPs are averaged over these grain boundary sectors. From Ref [38].

Figure 3: Heuristic multistage modeling framework for nucleation and growth of fatigue cracks. Cycles $\mathrm{N}_{\mathrm{MSC}}, \mathrm{N}_{\mathrm{PSC}}$, and $\mathrm{N}_{\mathrm{LC}}$ respectively represent the number of cycles to propagate the crack(s) in the regimes of microstructurally small crack growth (normally 3-10 times the grain or second phase size/spacing that affects retardation of the crack driving force, see inset figure from Kunkler et al. [52]), physically small crack (PSC) growth, and long crack (LC) growth. In the PSC regime, the crack is suitably long to be treated using conventional LEFM, but is still below the size considered amenable to a definition of the initial crack length for propagation analyses using LEFM [27]. See also Ref. [28].

Figure 4: Fatigue life prediction based on hierarchical multistage model ( $N=N_{\text {inc }}+N_{\text {prop, Msc }}$ ) for polycrystalline microstructure without inclusions (left). Variability in fatigue life prediction due to a single debonded near surface inclusion (right). Figures adopted from refs. [66] and [67], respectively. 